\title{
Modern contraceptive utilization and associated factors among reproductive-age women in Ethiopia: evidence from 2016 Ethiopia demographic and health survey
}

Mamo Nigatu Gebre* and Zerihun Kura Edossa

\begin{abstract}
Background: Modern contraceptive methods enable couples to enjoy sexual intercourse without fear of the risk of pregnancy at any desired time. The evidence from different studies done in Ethiopia on modern contraceptive method utilization was highly varied and not conclusive. Therefore, the current study aims to study the magnitude of modern contraceptive utilization and associated factors among reproductive-age women in Ethiopia based on 2016 EDHS data.

Method: A nationally representative 2016 EDHS data collected between January 18/ 2016 to June 27/2016 were used. Descriptive studies and logistic regression models were used to summarize descriptive data and measure statistical association respectively. Adjusted odds ratio and confidence interval were respectively used to measure association and its statistical significance. Finally, statistical significance was declared using a confidence interval.

Result: In the current study, the overall modern contraceptive utilization among reproductive-age women in Ethiopia was 3203 (20.42\%). The injectable contraceptive method was the most commonly used modern contraceptive method, 1886(58.88\%) followed by implant/Norplant, 779 (24.32\%). The results of multivariable logistic regression showed that age, residence, region, woman's occupation, number of living children, husband's education, age at first sexual intercourse, husband's desire for more children, wealth index and watching TV were independently associated to modern contraceptive utilization among reproductive-age women in Ethiopia.

Conclusions: The magnitude of modern contraceptive utilization among reproductive-age women in Ethiopia in the current study is unexpectedly low. Age, residence, region, woman's occupation, number of living children, husband's education, age at first sexual intercourse, husband's desire for more children, wealth index and watching TV were independent predictors of modern contraceptive use among reproductive-age women in Ethiopia. Any intervention strategy that promotes modern contraceptive method utilization should consider these factors for its better success.
\end{abstract}

Keywords: Modern contraceptive method, Reproductive age women, 2016 EDHS, Ethiopia

\footnotetext{
* Correspondence: mamogebre14@gmail.com

Department of Epidemiology, Faculty of Public Health, Institute of Health,

Jimma University, Jimma, Ethiopia

(c) The Author(s). 2020 Open Access This article is licensed under a Creative Commons Attribution 4.0 International License, which permits use, sharing, adaptation, distribution and reproduction in any medium or format, as long as you give appropriate credit to the original author(s) and the source, provide a link to the Creative Commons licence, and indicate if changes were made. The images or other third party material in this article are included in the article's Creative Commons licence, unless indicated otherwise in a credit line to the material. If material is not included in the article's Creative Commons licence and your intended use is not permitted by statutory regulation or exceeds the permitted use, you will need to obtain permission directly from the copyright holder. To view a copy of this licence, visit http://creativecommons.org/licenses/by/4.0/ The Creative Commons Public Domain Dedication waiver (http://creativecommons.org/publicdomain/zero/1.0/) applies to the data made available in this article, unless otherwise stated in a credit line to the data.
} 


\section{Background}

Access to safe and modern contraceptive methods contributes to more freedom, independence of women and gender equity and is, therefore, a cornerstone of women's rights and their sexual and reproductive health [1]. Contraceptive use helps couples and individuals realize their basic right to decide freely and responsibly when and how many children to have. The growing use of contraceptive methods has resulted in not only improvements in health-related outcomes such as reduced maternal mortality and infant mortality, but also improvements in schooling and economic outcomes, especially for girls and women [2].

In developing countries, two hundred twenty-two million women who want to delay or avoid pregnancy are not using any method of family planning [3]. Worldwide, the prevalence of unmet need among married or inunion women is as high as $10 \%$, whereas, it is doubled in the Africa region [2]. Ethiopia is still among countries with low contraceptive utilization rates even though considerable improvements have been made in the last decades [4].

Modern contraceptive methods enable couples to enjoy sex without fear of the risk of pregnancy at any desired time [5]. Modern contraceptive methods account for more than $90 \%$ of the contraceptive use worldwide. Globally in 2017, 58\% of married or in-union women of reproductive age were using a modern method which accounts for $92 \%$ of all contraceptive users [2].

Utilizing contraceptive methods prevent sexually transmitted diseases in addition to avoiding unwanted pregnancies, unintended birth, abortion, miscarriages and maternal death [3].

A community-based cross-sectional study done in Ho district of west Ghana showed that $89.8 \%$ of married women used modern contraceptives, and the injectable contraceptive method was the most used [6]. To the contradict, another study was done in the same country (Ghana) showed a prevalence of only $21 \%$ [7]. A community-based crosssectional study done on reproductive-age women in Yaoundé-Cameroon showed that 58.9\% used a modern contraceptive method [8]. Different studies done in Ethiopia [9-14] showed extremely variable results in the prevalence of modern contraceptive use which ranged from $20.8 \%$ from the study done at Bale Zone, South East Ethiopia [9] to $71.9 \%$ from the community-based cross-sectional study done at western Ethiopia [14]. Studies done in different parts of Ethiopia also showed that the injectable contraceptive method was the most used modern contraceptive method $[9,11,12,14]$.

Religious opposition, desire for more children, fear of side effects, husband opposition, inter-spousal discussion, Perceived husband approval, discussion with HEW, perceived cultural acceptability, Women's educational status, gravidity, postnatal care utilization, age, Husband's education, marital status, monthly income, fertility, media exposure, number of living children, woman's decision making autonomy, family planning counseling and Having postnatal care were some of the factors independently associated with modern contraceptive utilization [6, 8-15].

Evidence from the aforementioned studies done in Ethiopia was highly varied and the results are inconclusive. Therefore, the current study aims to study the magnitude of modern contraceptive utilization and its predictors among reproductive-age women in Ethiopia based on the 2016 EDHS data. The current study will provide up-to-date evidence for policymakers and other stakeholders working on family planning in Ethiopia and similar settings to solve problems related to modern contraceptive utilization based on evidence.

\section{Methods \\ Population}

All reproductive age women in Ethiopia, based on 2016 EDHS were included in the study.

\section{Data source}

The current study used data extracted from 2016 EDHS which was stratified into urban and rural areas and yielded twenty-one sampling strata. A two-stage sampling technique was done to select representative samples of independent enumeration areas in each stratum. In the first stage, 202 urban areas and 443 rural areas were selected using probability proportional to the size of enumeration area. Lists of households were prepared from the selected enumeration areas and served as a sampling frame for the selection of representative households in the second stage. Lastly, a total of 18,008 households were selected for the study out of which only 17 , 067 households were occupied. Complete interview was obtained from 16,650 households making the response rate $98 \%$. In those interviewed households, 16,583 women were identified and 15,683 women completed the interview making a response rate of $95 \%$. Therefore, data for the current study came from an individual record of 15,683 reproductive-age women. For all eligible women aged 15-49 years, the woman's questionnaire comprising five different parts including family planning was used to collect information [16].

\section{Statistical analysis}

Statistical package for social science (SPSS) was used for statistical analysis. Descriptive studies like measures of central tendency and measures of dispersion for continuous data, and frequency count and proportion for categorical data were used to summarize descriptive data. Bivariate logistic regression was used to select 
candidate variables for multivariable logistic regression. In the bivariate logistic regression, a $p$-value of less than 0.2 was used as a cut of point. Variable inflation factor (VIF) was used to check multi-collinearities between candidate variables before fitting the final model. Multivariable logistic regression was used to identify independent predictors of modern contraceptive method utilization among reproductive-age women in Ethiopia and to control confounders. Adjusted odds ratio and confidence interval $(\mathrm{CI})$ were respectively used to measure the association between modern contraceptive utilization and predictor variables and their statistical significance in the final model. The confidence interval was used to declare statistical significance in the final model. Hosmer and Lameshow test was used to check model fitness.

\section{Operational definitions}

Modern contraceptive utilization: in the current study a woman was considered as modern contraceptive method utilizer if she had been using at least one of the modern contraceptives (female sterilization, male sterilization, IUCD, injectable, implants, pills, male condom, female condom, emergency contraception, and standard days method) during EDHS data collection period.

Modern contraceptive non-utilization: a woman was considered to be non-utilizer of the modern contraceptive method if she had been using traditional methods like rhythm method, lactation amenorrhea method, and withdrawal or if she had not been using any type of contraception during EDHS data collection period.

\section{Results}

Socio-demographic and socio-economic characteristics In the interviewed households, 16,583 eligible women were identified for individual interviews. Interviews were completed with 15,683 women, yielding a response rate of $95 \%$. The mean age of respondents was 27.94 ( \pm 9.16$)$ years with the age range of 34 years. From the total 15, 683 respondents interviewed, 3498 (22.3\%) were within the age group of 15-19 years. Out of the total reproductive age women interviewed, $18.51 \%$ (2903) and $18.14 \%$ (2845) were found in the age groups of 25-29 and 3034 years respectively (Fig. 1). From the total respondents, $1892(12.06 \%)$ and 907 (5.78\%) were from Oromiya and Harari region respectively (Fig. 2). Regarding the occupation of the participants, $8045(51.3 \%)$ were not working and 211(1.3\%) were clericals (Fig. 3). Regarding the residence of respondents, 10,335 (65.9\%) women were rural residents. Concerning educational status, 7033(44.8\%) were not educated and 4524 (46.1\%) women had uneducated husbands. 7591 (63.5\%) and 4094 (39.9\%) reproductive age women committed first sexual intercourse and bore the first child at age of less than 18 years respectively. Regarding the economic status of the women, 5699(36.3\%) women were from the richest family. Concerning decision making on contraceptive use, 2104 (70.6\%) reproductive age women jointly decided with their husbands (Table 1).

\section{Prevalence of modern contraceptive use}

The overall prevalence of modern contraceptive utilization was 3203 (20.42\%). The injectable contraceptive method was the most, $1886(58.88 \%)$ commonly used modern contraceptive method followed by implant/Norplant, 779 (24.32\%) (Fig. 4). From the reproductive age women aged $15-19$ years only $5.91 \%$ (207) utilized modern contraceptive method whereas 28.64 and $28.56 \%$ of reproductive age women aged between 25 and 29 years and $30-34$ years utilized modern contraceptive methods respectively. There was also a disparity of modern contraceptive utilization between rural and urban resident reproductive-age women; only 1946(18.8\%) rural residents utilized modern contraceptive whereas 1257 (23.5\%) urban residents utilized modern contraceptive. Modern contraceptive utilization was also highly varied based on marital status; only 119 (2.78\%) unmarried reproductive age women utilized modern contraceptive methods whereas 2886 (29.3\%) reproductive-age women who were married and living together utilized modern

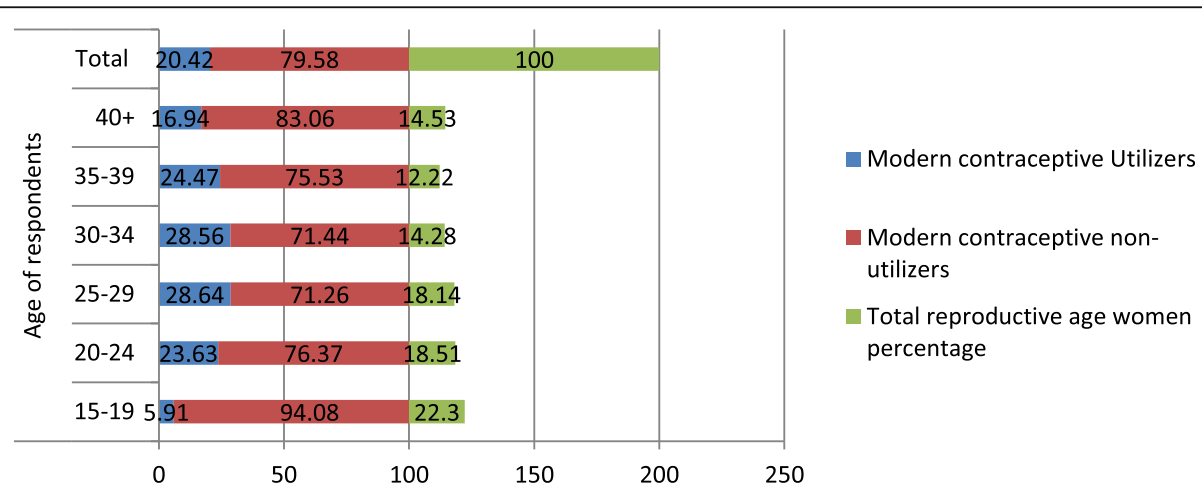

Fig. 1 Total reproductive age women and Modern contraceptive utilization by age, 2016 


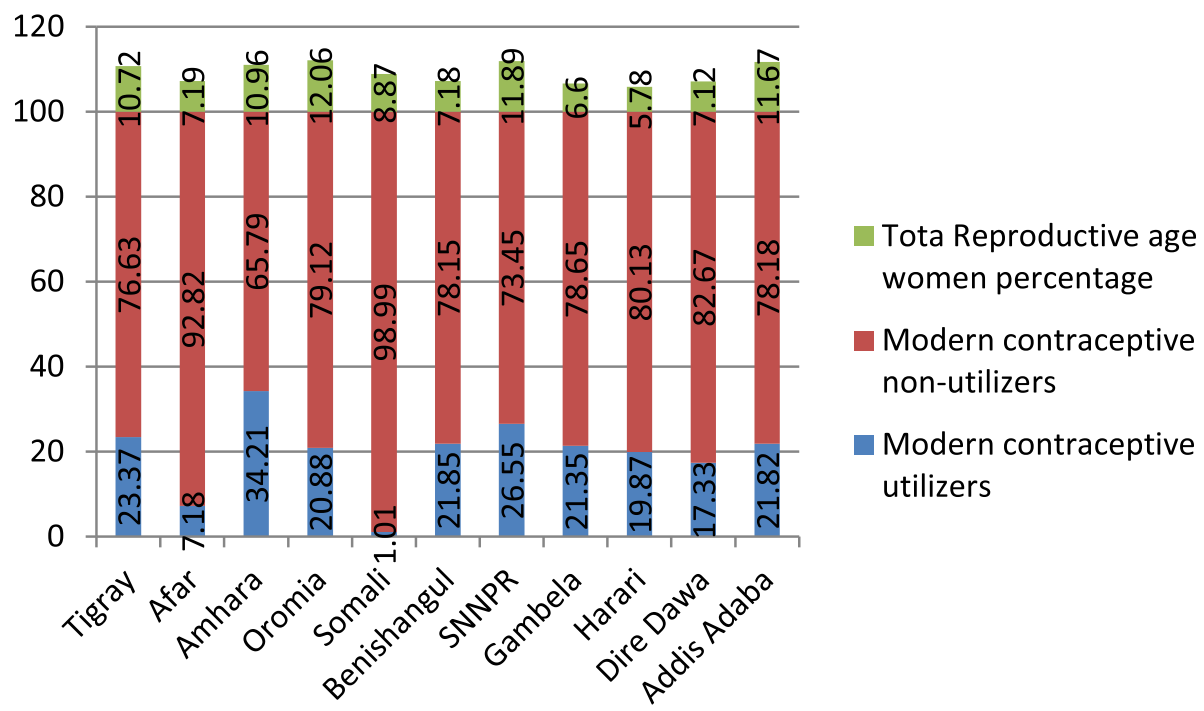

Fig. 2 Total reproductive age women and Modern contraceptive utilization by regions of Ethiopia, 2016

contraceptive method. There was also a great disparity in modern contraceptive utilization based on respondents' wealth index; only 198(12.5\%) reproductive women from poorest households utilized modern contraceptive methods whereas 538 (26.3\%) women from richer households utilized modern contraceptive method. Modern contraceptive utilization was also highly varied by regions of Ethiopia; 34.21 and $1.01 \%$ reproductive age women utilized modern contraceptive methods from Amhara and Harari regions respectively.

\section{Individual and behavioral characteristics of reproductive age women in Ethiopia}

Regarding age at first sexual intercourse and age at first childbirth, 7591 (63.5\%) and 5286(51.5\%) had committed their first sexual intercourse and had born their first child at age of less than 18 years and 18-24 year respectively. 5409 (34.5\%) and 5539(38.4\%) reproductive age women did not bear a child and did not have a living child respectively. 13,106(83.6\%), 10,338(65.9\%) and 10, 084(64.3\%) did not read the newspaper, listen to the radio and watch television. Regarding decision making on contraceptive use, $2104(70.6 \%)$ women made the decision jointly with their husbands (Table 2).

\section{Results of bivariate analysis}

Bivariate logistic regression was fitted to identify candidate variables for multivariable logistic regression. Accordingly: Age of respondent, residence, region of residence, current marital status, educational level,

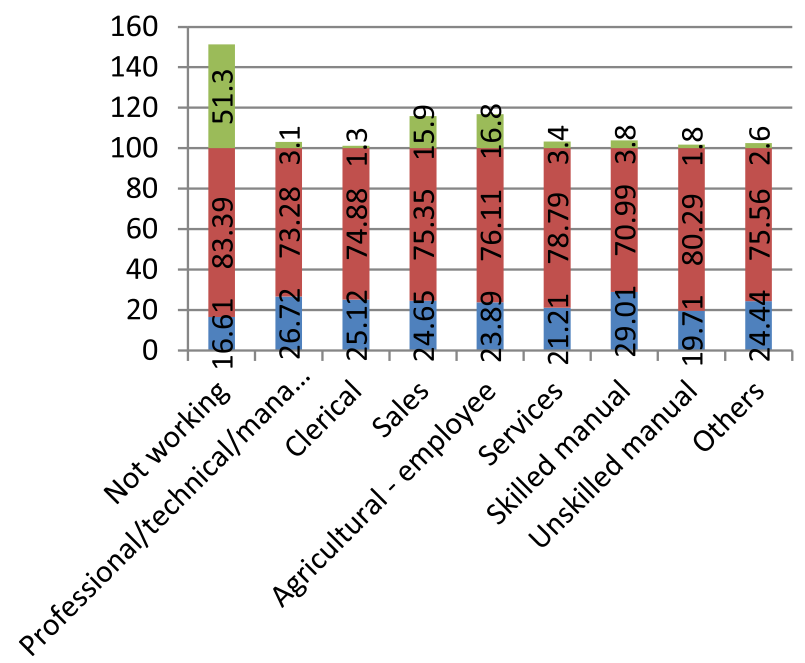

Reproductive age women percentage

Modern contraceptive non-utilizers

- Moder contraceptive utilizers

Fig. 3 Reproductive age women percentage and modern contraceptive method utilization by occupation, 2016 
Table 1 Socio-demographic and socio-economic characteristics of reproductive age women in Ethiopia, 2016

\begin{tabular}{|c|c|c|c|c|}
\hline \multirow{2}{*}{$\begin{array}{l}\text { Socio-demographic and } \\
\text { socio-economic characteristics }\end{array}$} & \multirow[t]{2}{*}{ Variable Categories } & \multirow{2}{*}{$\begin{array}{l}\text { Frequency } \\
\text { (\%) }\end{array}$} & \multicolumn{2}{|c|}{ Modern contraceptive utilization } \\
\hline & & & Yes (\%) & No (\%) \\
\hline \multirow[t]{2}{*}{ Residence } & Urban & $5348(34.1)$ & $1257(23.5)$ & $4091(76.5)$ \\
\hline & rural & $10,335(65.9)$ & $1946(18.8)$ & $8389(81.2)$ \\
\hline \multirow[t]{3}{*}{ current marital status } & never married & $4278(27.3)$ & $119(2.78)$ & $4159(97.2)$ \\
\hline & married/living together & $9824(62.6)$ & $2886(29.3)$ & $6938(70.7)$ \\
\hline & married but not together & $1581(10.1)$ & $198(12.5)$ & $1383(87.5)$ \\
\hline \multirow[t]{4}{*}{ Respondents educational level } & No education & $7033(44.8)$ & $1325(18.83)$ & $5708(81.16)$ \\
\hline & Primary & $5213(33.2)$ & $1149(22.04)$ & $4064(77.96)$ \\
\hline & Secondary & $2238(14.3)$ & $428(19.12)$ & $1810(80.88)$ \\
\hline & Higher & $1199(7.6)$ & $301(25.10)$ & $898(74.90)$ \\
\hline \multirow[t]{4}{*}{ Husband's education } & not educated & $4524(46.1)$ & $905(20.0)$ & $3619(80.0)$ \\
\hline & primary & 3054 (31.1) & $1121(36.7)$ & $1933(63.3)$ \\
\hline & secondary & $1226(12.5)$ & $470(38.3)$ & $756(61.7)$ \\
\hline & higher & $1020(10.4)$ & $390(38.3)$ & $630(61.7)$ \\
\hline \multirow[t]{5}{*}{ Wealth index } & Poorest & 3894 (24.8) & $327(8.4)$ & 3567 (91.6) \\
\hline & Poorer & $2046(13.1)$ & $432(21.1)$ & 1614 (78.9) \\
\hline & Middle & $2002(12.8)$ & $494(24.7)$ & 1508 (75.3) \\
\hline & Richer & $2042(13.0)$ & $538(26.3)$ & 1504 (73.7) \\
\hline & Richest & 5699 (36.3) & $1412(24.8)$ & $4287(75.2)$ \\
\hline
\end{tabular}

occupation, total children ever born, number of living children, husband's educational level, age at first sex, husband's desire for child, wealth index, frequency of listening to radio and frequency of watching television were candidate for multivariable logistic regression (Table 3).

\section{Predictors of modern contraceptive use among reproductive-age women in Ethiopia, 2016}

A multivariable logistic regression model was fitted to identify independent predictors of modern contraceptive method utilization among reproductive-age women in Ethiopia. Accordingly; age, place of residence, region of residence, occupation, number of living children, Husband's education, age at first sex, husband's desire for children, wealth index and frequency of watching television were independently associated with modern contraceptive method utilization.

Woman whose age was between 15 and 19, 20-24, $25-29,30-34$ and $35-39$ was nearly 1.9 [AOR = 1.911(1.301,2.806)], $2.4[$ AOR $=2.389(1.853,3.081)], 2.2$ $[2.196(1.782,2.706)], 1.9[\mathrm{AOR}=1.938(1.589,2.363)]$ and $1.8[1.797(1.464,2.207)]$ times more likely to utilize modern contraceptive method as compared to woman whose age was 40 year and above.

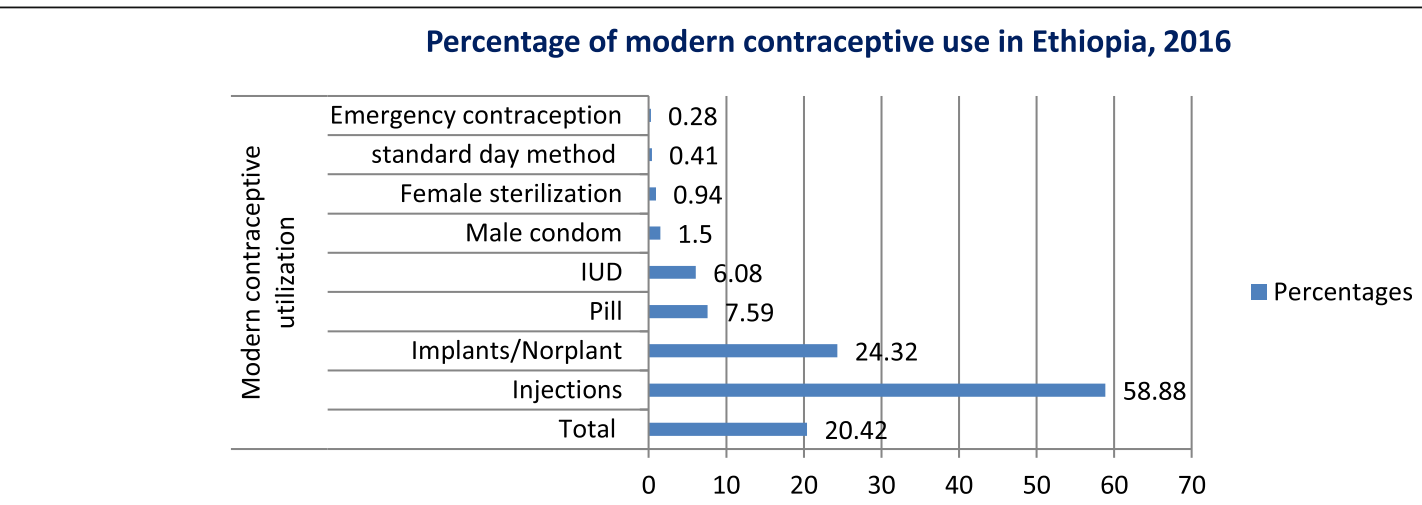

Fig. 4 Prevalence of Modern Contraceptive Utilization in Ethiopia, 2016 
Table 2 Individual and behavioral related characteristics of reproductive age women in Ethiopia, 2016

\begin{tabular}{|c|c|c|c|c|}
\hline \multirow[t]{2}{*}{ Variable } & \multirow[t]{2}{*}{ Categories } & \multirow{2}{*}{$\begin{array}{l}\text { Frequency } \\
\text { (\%) }\end{array}$} & \multicolumn{2}{|c|}{ Modern contraceptive utilization } \\
\hline & & & Yes & No \\
\hline \multirow[t]{3}{*}{ Age at first sex } & less 18 years & $7591(63.5)$ & $1989(26.2)$ & $5602(73.8)$ \\
\hline & $18-24$ & $3961(33.1)$ & $1125(28.4)$ & $2836(71.6)$ \\
\hline & 25 and above & $410(3.4)$ & $77(18.8)$ & $333(81.2)$ \\
\hline \multirow[t]{3}{*}{ Age at first childbirth } & less 18 years & $4094(39.9)$ & $1044(25.5)$ & $3050(74.5)$ \\
\hline & $18-24$ & $5286(51.5)$ & $1549(29.3)$ & $3737(70.7)$ \\
\hline & 25 and above & $886(8.6)$ & $235(26.5)$ & $651(73.5)$ \\
\hline \multirow[t]{4}{*}{ Total children ever born } & No child & $5409(34.5)$ & $374(6.91)$ & 5035 (93.09) \\
\hline & $1-2$ & $3678(23.5)$ & $1266(34.42)$ & $2412(65.58)$ \\
\hline & $3-4$ & $2683(17.1)$ & $785(29.26)$ & $1898(70.74)$ \\
\hline & 5 and above & $3913(25.0)$ & 778 (19.88) & $3135(80.12)$ \\
\hline \multirow[t]{4}{*}{ Number of living children } & No child & $5539(38.4)$ & $390(7.04)$ & $5149(92.96)$ \\
\hline & $1-2$ & $3983(27.6)$ & $1355(34.02)$ & $2628(65.98)$ \\
\hline & $3-4$ & $2930(20.2)$ & $807(27.54)$ & $2123(72.46)$ \\
\hline & 5 and above & $1990(13.8)$ & $438(22.01)$ & $1552(77.10)$ \\
\hline \multirow[t]{3}{*}{ Frequency of reading newspaper or magazine } & Not at all & $13,106(83.6)$ & $2632(20.08)$ & $10,474(79.92)$ \\
\hline & Less than once a week & $1881(12.0)$ & $428(22.75)$ & $1453(77.25)$ \\
\hline & At least once a week & $696(4.4)$ & $143(20.55)$ & $553(79.45)$ \\
\hline \multirow[t]{3}{*}{ Frequency of listening to the radio } & Not at all & $10,338(65.9)$ & $1908(18.46)$ & $8430(81.54)$ \\
\hline & Less than once a week & $2644(16.9)$ & $623(23.56)$ & $2021(76.44)$ \\
\hline & At least once a week & $2701(17.1)$ & $672(24.88)$ & $2029(78.12)$ \\
\hline \multirow[t]{3}{*}{ Frequency of watching TV } & Not at all & $10,084(64.3)$ & $1820(18.05)$ & $8264(81.95)$ \\
\hline & Less than once a week & $1780(11.3)$ & $444(24.94)$ & $1336(75.06)$ \\
\hline & At least once a week & $3819(24.4)$ & 939 (24.59) & $2880(75.41)$ \\
\hline \multirow[t]{3}{*}{ Decision make on contraceptive use } & Mainly respondent & $726(24.4)$ & $705(97.1)$ & $21(2.9)$ \\
\hline & Mainly husband, partner & $150(5.0)$ & $147(98.0)$ & $3(2.0)$ \\
\hline & Joint decision & 2104 (70.6) & 2034 (96.7) & $70(3.3)$ \\
\hline
\end{tabular}

The odds of modern contraceptive utilization among urban resident women was nearly 1.5 [AOR $=1.5129$ $(1.204,1.900)]$ times more likely than the odds of modern contraceptive use among rural resident woman.

A Women who resides in Tigray, Amhara and SNNPR regions of Ethiopia was nearly $1.4[\mathrm{AOR}=1.370$ (1.041, $1.804)], 3[\mathrm{AOR}=2.890(2.181,3.831)]$ and $2[\mathrm{AOR}=1.95$ $(1.484,2.565)]$ times more likely to utilize modern contraceptive method as compared to woman who resides in Addis Ababa respectively. A woman who resides in Afar and Somali region of Ethiopia was nearly 55\% [(AOR $=.446$ $(.310, .643)]$ and $93 \%[(\mathrm{AOR}=.070,(.038, .130)]$ times less likely to utilize modern contraceptive method as compared to a woman who resides in Addis Ababa respectively.

A woman who earns her life by selling goods, agricultural employment, skilled manual working, and other different jobs was nearly $1.3[\mathrm{AOR}=1.336(1.136,1.572)]$, $1.2[1.192(1.022,1.390)], \quad 1.4[\mathrm{AOR}=1.425(1.085,1.872)]$ and $1.6[\mathrm{AOR}=1.331(1.039,1.706)]$ times more likely to utilize modern contraceptive method as compared to a woman who did not have any job respectively.

A woman who had no child or who had 1-2 child/ children was nearly $75 \%$ [AOR $=.255(.117, .556)]$ and $34 \%[\mathrm{AOR}=.658(.455, .952)]$ times less likely to utilize modern contraceptive methods as compared to a woman who had born 5 or more children.

A woman whose husband had completed primary education was nearly $1.4[\mathrm{AOR}=1.347(1.173,1.546)]$ times more likely to utilize modern contraceptive methods as compared to a woman whose husband was not educated.

A woman who had started her first sexual intercourse at an age of less than 18 years was nearly 1.6 [AOR = $1.582(1.054,2.373)]$ times more likely to utilize modern contraceptive methods as compared to a woman who started her first sexual intercourse at an age of 25 or more year respectively.

The odds of modern contraceptive utilization among women whose husbands want more children was nearly 
Table 3 Bivariate analysis of the association between modern contraceptive utilization and associated factors

\begin{tabular}{|c|c|c|c|c|c|c|}
\hline \multirow[t]{2}{*}{ Characteristics } & \multirow[t]{2}{*}{ Category } & \multirow[t]{2}{*}{$\begin{array}{l}\text { Frequency } \\
(\%)\end{array}$} & \multicolumn{2}{|c|}{$\begin{array}{l}\text { Modern contraceptive } \\
\text { utilization }\end{array}$} & \multirow[t]{2}{*}{ COR $(95 \% \mathrm{Cl})$} & \multirow[t]{2}{*}{$P$} \\
\hline & & & Yes & No & & \\
\hline \multirow[t]{6}{*}{ Age of respondent } & $15-19$ & $3498(22.3)$ & 207 & 3291 & $.308(.258,369)$ & $.000^{*}$ \\
\hline & $20-24$ & $2903(18.5)$ & 686 & 2217 & $1.517(1.321,1.744)$ & $.000^{*}$ \\
\hline & $25-29$ & $2845(18.1)$ & 815 & 2030 & $1.969(1.718,2.256)$ & $.000^{*}$ \\
\hline & $30-34$ & $2241(14.3)$ & 640 & 1601 & $1.960(1.700,2.261)$ & $.000^{*}$ \\
\hline & $35-39$ & $1917(12.2)$ & 469 & 1448 & $1.588(1.366,1.847)$ & $.000^{*}$ \\
\hline & $40^{+}$ & $2279(14.5)$ & 386 & 1893 & 1 & \\
\hline \multirow[t]{2}{*}{ Residence } & Urban & $5348(34.1)$ & 1257 & 4091 & $1.325(1.223,1.435)$ & $.000^{*}$ \\
\hline & rural & $10,335(65.9)$ & 1946 & 8389 & 1 & \\
\hline \multirow[t]{11}{*}{ Region } & Tigray & $1682(10.7)$ & 393 & 1289 & $1.092(.932,1.280)$ & .274 \\
\hline & Afar & $1128(7.2)$ & 81 & 1047 & $.277(.215,357)$ & $.000^{*}$ \\
\hline & Amhara & $1719(11.0)$ & 588 & 1131 & $1.863(1.604,2.163)$ & $.000^{*}$ \\
\hline & Oromia & $1892(12.1)$ & 395 & 1497 & $.945(.808,1.106)$ & .483 \\
\hline & Somali & $1391(8.9)$ & 14 & 1377 & $.036(.021, .062)$ & $.000^{*}$ \\
\hline & Benishangul & $1126(7.2)$ & 246 & 880 & $1.002(.837,1.199)$ & .986 \\
\hline & SNNPR & 1849 (11.8) & 491 & 1358 & $1.295(1.113,1.508)$ & $.001^{*}$ \\
\hline & Gambela & $1035(6.6)$ & 221 & 814 & $.973(.808,1.171)$ & .771 \\
\hline & Harari & $906(5.8)$ & 180 & 726 & $.888(.729,1.082)$ & .240 \\
\hline & Dire Dawa & $1131(7.2)$ & 196 & 935 & $.751(.621, .908)$ & $.003^{*}$ \\
\hline & Addis Adaba & $1824(11.6)$ & 398 & 1426 & 1 & \\
\hline \multirow[t]{3}{*}{ current marital status } & never married & $4278(27.3)$ & 119 & 4159 & 1 & \\
\hline & married/living together & $9824(62.6)$ & 2886 & 6938 & $.200(.158,253)$ & $.000^{*}$ \\
\hline & married but not together & $1581(10.1)$ & 198 & 1383 & $2.905(2.488,3.393)$ & $.000^{*}$ \\
\hline \multirow[t]{4}{*}{ Respondent educational level } & No education & $7033(44.8)$ & 1325 & 5708 & 1 & \\
\hline & Primary & $5213(33.2)$ & 1149 & 4064 & $.693(.600, .799)$ & $.000^{*}$ \\
\hline & Secondary & $2238(14.3)$ & 428 & 1810 & $.843(.729, .976)$ & $.022^{*}$ \\
\hline & Higher & $1199(7.6)$ & 301 & 898 & $.705(.597, .834)$ & $.000^{*}$ \\
\hline \multirow[t]{9}{*}{ Respondent's Occupation } & Not working & $8045(51.3)$ & 1336 & 6709 & 1 & \\
\hline & Professional/technical/managerial & $494(3.1)$ & 132 & 362 & $.616(.487, .779)$ & $.000^{*}$ \\
\hline & Clerical & $211(1.3)$ & 53 & 158 & $1.127(.833,1.526)$ & .437 \\
\hline & Sales & $2495(15.9)$ & 615 & 1880 & $1.037(.705,1.525)$ & .853 \\
\hline & Agricultural - employee & $2637(16.8)$ & 630 & 2007 & $1.011(.791,1.293)$ & .928 \\
\hline & Services & $528(3.4)$ & 112 & 416 & $.971(.760,1.240)$ & .811 \\
\hline & Skilled manual & $593(3.8)$ & 172 & 421 & $.832(.611,1.134)$ & .244 \\
\hline & Unskilled manual & $279(1.8)$ & 55 & 224 & $1.263(.946,1.686)$ & $.113^{*}$ \\
\hline & Others & $401(2.6)$ & 98 & 303 & $.759(.523,1.102)$ & $.147^{*}$ \\
\hline \multirow[t]{4}{*}{ Total children ever born } & No child & $5409(34.5)$ & 374 & 5035 & $.299(.263,341)$ & \\
\hline & $1-2$ & $3678(23.5)$ & 1266 & 2412 & $2.115(1.906,2.347)$ & $.000^{*}$ \\
\hline & $3-4$ & $2683(17.1)$ & 785 & 1898 & $1.667(1.486,1.869)$ & $.000^{*}$ \\
\hline & 5 and above & $3913(25.0)$ & 778 & 3135 & 1 & $.000^{*}$ \\
\hline \multirow[t]{3}{*}{ Number of living children } & No child & $5539(38.4)$ & 390 & 5149 & $.268(.232, .311)$ & \\
\hline & $1-2$ & $3983(27.6)$ & 1355 & 2628 & $1.827(1.613,2.070)$ & $.000^{*}$ \\
\hline & $3-4$ & $2930(20.2)$ & 807 & 2123 & $1.347(1.179,1.539)$ & $.000^{*}$ \\
\hline
\end{tabular}


Table 3 Bivariate analysis of the association between modern contraceptive utilization and associated factors (Continued)

\begin{tabular}{|c|c|c|c|c|c|c|}
\hline \multirow[t]{2}{*}{ Characteristics } & \multirow[t]{2}{*}{ Category } & \multirow[t]{2}{*}{$\begin{array}{l}\text { Frequency } \\
\text { (\%) }\end{array}$} & \multicolumn{2}{|c|}{$\begin{array}{l}\text { Modern contraceptive } \\
\text { utilization }\end{array}$} & \multirow[t]{2}{*}{ COR $(95 \% \mathrm{Cl})$} & \multirow[t]{2}{*}{$P$} \\
\hline & & & Yes & No & & \\
\hline & 5 and above & 1990 (13.8) & 438 & 1552 & 1 & $.000^{*}$ \\
\hline \multirow[t]{4}{*}{ Husband's education } & not educated & $4524(46.1)$ & 905 & 3619 & 1 & \\
\hline & primary & 3054 (31.1) & 1121 & 1933 & $.404(.349, .467)$ & $.000^{*}$ \\
\hline & secondary & $1226(12.5)$ & 470 & 756 & $.937(.809,1.084)$ & .381 \\
\hline & higher & $1020(10.4)$ & 390 & 630 & $1.004(.847,1.191)$ & .961 \\
\hline \multirow[t]{3}{*}{ Age at first sex } & less 18 years & $7591(63.5)$ & 1989 & 5602 & $1.535(1.192,1.978)$ & $.000^{*}$ \\
\hline & $18-24$ & 3961 (33.1) & 1125 & 2836 & $1.716(1.326,2.219)$ & $.000^{*}$ \\
\hline & 25 and above & $410(3.4)$ & 77 & 333 & 1 & \\
\hline \multirow[t]{3}{*}{ Age at first childbirth } & less 18 years & 4094 (39.9) & 1044 & 3050 & $.948(.804,1.118)$ & .527 \\
\hline & $18-24$ & $5286(51.5)$ & 1549 & 3737 & $1.148(.978,1.348)$ & $.091^{*}$ \\
\hline & 25 and above & $886(8.6)$ & 235 & 651 & 1 & \\
\hline \multirow[t]{4}{*}{ Husband's desire for children } & Both want the same & 3894 (39.8) & 1364 & 2530 & 1 & $.000^{*}$ \\
\hline & Husband wants more & $2641(27.0)$ & 610 & 2031 & $1.612(1.444,1.800)$ & .456 \\
\hline & Husband wants fewer & $634(6.5)$ & 225 & 409 & $.898(.792,1.019)$ & $.000^{*}$ \\
\hline & Don't know & $2626(26.8)$ & 658 & 1968 & $1.645(1.367,1.980)$ & \\
\hline \multirow[t]{5}{*}{ Wealth index } & Poorest & $3894(24.8)$ & 327 & 3567 & 1 & \\
\hline & Poorer & $2046(13.1)$ & 432 & 1614 & $.278(.245,316)$ & $.000^{*}$ \\
\hline & Middle & $2002(12.8)$ & 494 & 1508 & $.813(.719, .918)$ & $.001^{*}$ \\
\hline & Richer & $2042(13.0)$ & 538 & 1504 & $.995(.884)$, & .928 \\
\hline & Richest & $5699(36.3)$ & 1412 & 4287 & $1.086(.968,1.219)$ & $.161^{*}$ \\
\hline \multirow[t]{3}{*}{ The decision makes on contraceptive use } & Mainly respondent & $726(24.4)$ & 705 & 21 & $1.155(.704,1.896)$ & .568 \\
\hline & Mainly husband, partner & $150(5.0)$ & 147 & 3 & $1.686(.525,5.421)$ & .380 \\
\hline & Joint decision & $2104(70.6)$ & 2034 & 70 & 1 & \\
\hline \multirow[t]{3}{*}{ Frequency of reading newspaper or magazine } & Not at all & $13,106(83.6)$ & 2632 & 10,474 & 1 & \\
\hline & Less than once a week & $1881(12.0)$ & 428 & 1453 & $.972(.805,1.174)$ & .766 \\
\hline & At least once a week & $696(4.4)$ & 143 & 553 & $1.139(.920,1.410)$ & .231 \\
\hline \multirow[t]{3}{*}{ Frequency of listening to the radio } & Not at all & $10,338(65.9)$ & 1908 & 8430 & 1 & \\
\hline & Less than once a week & $2644(16.9)$ & 623 & 2021 & $.683(.618, .756)$ & $.000^{*}$ \\
\hline & At least once a week & $2701(17.1)$ & 672 & 2029 & $.931(.821,1.055)$ & .261 \\
\hline \multirow[t]{3}{*}{ Frequency of watching TV } & Not at all & $10,084(64.3)$ & 1820 & 8264 & 1 & \\
\hline & Less than once a week & $1780(11.3)$ & 444 & 1336 & $.675(.618, .739)$ & $.000^{*}$ \\
\hline & At least once a week & $3819(24.4)$ & 939 & 2880 & $1.019(.895,1.161)$ & .72 \\
\hline
\end{tabular}

*Statistically significant

$25 \%[\mathrm{AOR}=.749(.653, .860)]$ times less likely than the odds of modern contraceptive utilization among women who want the same number of children with their husbands. The odds of modern contraceptive utilization among women where neither husbands' nor women's desire for more children is not known was nearly $32 \%[\mathrm{AOR}=.677(.590, .776)]$ times less likely than the odds of modern contraceptive utilization among women where women and husbands want the same number of children.
A woman who was from the richest, a richer, middle and poorer household was nearly $3.5[\mathrm{AOR}=3.462(2$. $672,4.485], 3[\mathrm{AOR}=3.034(2.471,3.726)], 2.5[\mathrm{AOR}=$ $2.471(2.021,3.022)]$ and $2[\mathrm{AOR}=1.929(1.580,2.356)]$ times more likely to utilize modern contraceptive method as compared to reproductive age woman from poorest household respectively.

A woman who watches television at least once a week and less than once a week was nearly $1.3[\mathrm{AOR}=$ $1.259(1.036,1.531)]$ and $1.4[\mathrm{AOR}=1.368(1.110,1.687)]$ 
Table 4 Independent predictors of modern contraceptive utilization among reproductive-age women in Ethiopia, 2016

\begin{tabular}{|c|c|c|c|c|}
\hline Characteristics & Category & Frequency (\%) & COR $(95 \% \mathrm{Cl})$ & AOR $(95 \% \mathrm{Cl})$ \\
\hline \multirow[t]{6}{*}{ Age of respondent } & $15-19$ & $3498(22.3)$ & $.308(.258,369)$ & $1.911(1.301,2.806)^{*}$ \\
\hline & $20-24$ & $2903(18.5)$ & $1.517(1.321,1.744)$ & $2.389(1.853,3.081) *$ \\
\hline & $25-29$ & $2845(18.1)$ & $1.969(1.718,2.256)$ & $2.196(1.782,2.706) *$ \\
\hline & $30-34$ & $2241(14.3)$ & $1.960(1.700,2.261)$ & $1.938(1.589,2.363)$ * \\
\hline & $35-39$ & 1917 (12.2) & $1.588(1.366,1.847)$ & $1.797(1.464,2.207)$ * \\
\hline & $40^{+}$ & $2279(14.5)$ & 1 & 1 \\
\hline \multirow[t]{2}{*}{ Residence } & Urban & $5348(34.1)$ & $1.325(1.223,1.435)$ & $1.512(1.204,1.900) *$ \\
\hline & rural & $10,335(65.9)$ & 1 & 1 \\
\hline \multirow[t]{11}{*}{ Region } & Tigray & $1682(10.7)$ & $1.092(.932,1.280)$ & $1.370(1.041,1.804) *$ \\
\hline & Afar & $1128(7.2)$ & $.277(.215,357)$ & $.446(.310, .643) *$ \\
\hline & Amhara & $1719(11.0)$ & $1.863(1.604,2.163)$ & $2.890(2.181,3.831) *$ \\
\hline & Oromia & $1892(12.1)$ & $.945(.808,1.106)$ & $1.294(.986,1.699)$ \\
\hline & Somali & $1391(8.9)$ & $.036(.021, .062) *$ & $.070(.038, .130) *$ \\
\hline & Benishangul & $1126(7.2)$ & $1.002(.837,1.199)$ & $1.252(.925,1.694)$ \\
\hline & SNNPR & 1849 (11.8) & $1.295(1.113,1.508)$ & $1.951(1.484,2.565)$ * \\
\hline & Gambela & $1035(6.6)$ & $.973(.808,1.171)$ & $1.103(.824,1.478)$ \\
\hline & Harari & $906(5.8)$ & $.888(.729,1.082)$ & $.777(.584,1.034)$ \\
\hline & Dire Dawa & $1131(7.2)$ & $.751(.621, .908)$ & $.761(.573,1.011)$ \\
\hline & Addis Adaba & $1824(11.6)$ & 1 & 1 \\
\hline \multirow[t]{9}{*}{ Respondent's Occupation } & Not working & $8045(51.3)$ & 1 & 1 \\
\hline & Professional/technical/managerial & $494(3.1)$ & $.616(.487, .779)$ & $1.095(.745,1.608)$ \\
\hline & Clerical & $211(1.3)$ & $1.127(.833,1.526)$ & $1.287(.739,2.240)$ \\
\hline & Sales & $2495(15.9)$ & $1.037(.705,1.525)$ & $1.336(1.136,1.572) *$ \\
\hline & Agricultural - employee & $2637(16.8)$ & $1.011(.791,1.293)$ & $1.192(1.022,1.390) *$ \\
\hline & Services & $528(3.4)$ & $.971(.760,1.240)$ & $1.229(.868,1.740)$ \\
\hline & Skilled manual & $593(3.8)$ & $.832(.611,1.134)$ & $1.425(1.085,1.872) *$ \\
\hline & Unskilled manual & $279(1.8)$ & $1.263(.946,1.686)$ & $1.382(.858,2.227)$ \\
\hline & Others & $401(2.6)$ & $.759(.523,1.102)$ & $1.570(1.085,2.271) *$ \\
\hline \multirow[t]{4}{*}{ Total children ever born } & No child & $5409(34.5)$ & $.299(.263,341)$ & $1.711(1.168,2.505) *$ \\
\hline & $1-2$ & $3678(23.5)$ & $2.115(1.906,2.347)$ & $1.331(1.039,1.706) *$ \\
\hline & $3-4$ & $2683(17.1)$ & $1.667(1.486,1.869)$ & $1.421(1.123,2.341) *$ \\
\hline & 5 and above & $3913(25.0)$ & 1 & 1 \\
\hline \multirow[t]{4}{*}{ Number of living children } & No child & $5539(38.4)$ & $.268(.232, .311)$ & $.255(.117, .556) *$ \\
\hline & $1-2$ & $3983(27.6)$ & $1.827(1.613,2.070)$ & $.658(.455, .952) *$ \\
\hline & $3-4$ & $2930(20.2)$ & $1.347(1.179,1.539)$ & $.792(.616,1.018)$ \\
\hline & 5 and above & $1990(13.8)$ & 1 & 1 \\
\hline \multirow[t]{4}{*}{ Husband's education } & not educated & $4524(46.1)$ & 1 & 1 \\
\hline & primary & $3054(31.1)$ & $.404(.349, .467)$ & $1.347(1.173,1.546) *$ \\
\hline & secondary & $1226(12.5)$ & $.937(.809,1.084)$ & $1.069(.872,1.309)$ \\
\hline & higher & $1020(10.4)$ & $1.004(.847,1.191)$ & $.956(.749,1.221)$ \\
\hline \multirow[t]{3}{*}{ Age at firs sex } & less 18 year & $7591(63.5)$ & $1.535(1.192,1.978)$ & $1.582(1.054,2.373) *$ \\
\hline & 18-24 year & $3961(33.1)$ & $1.716(1.326,2.219)$ & $1.428(.964,2116)$ \\
\hline & 25 and above & $410(3.4)$ & 1 & 1 \\
\hline Husband's desire for children & Both want the same & 3894 (39.8) & 1 & 1 \\
\hline
\end{tabular}


Table 4 Independent predictors of modern contraceptive utilization among reproductive-age women in Ethiopia, 2016 (Continued)

\begin{tabular}{|c|c|c|c|c|}
\hline Characteristics & Category & Frequency (\%) & COR $(95 \% \mathrm{Cl})$ & AOR $(95 \% \mathrm{Cl})$ \\
\hline & Husband wants more & $2641(27.0)$ & $1.612(1.444,1.800)$ & $.749(.653, .860) *$ \\
\hline & Husband wants fewer & $634(6.5)$ & $.898(.792,1.019)$ & $.949(.770,1.170)$ \\
\hline & Don't know & $2626(26.8)$ & $1.645(1.367,1.980)$ & $.677(.590, .776) *$ \\
\hline \multirow[t]{5}{*}{ Wealth index } & Poorest & 3894 (24.8) & 1 & 1 \\
\hline & Poorer & $2046(13.1)$ & $.278(.245, .316)$ & $1.929(1.580,2.356)$ * \\
\hline & Middle & $2002(12.8)$ & $.813(.719, .918)$ & $2.471(2.021,3.022) *$ \\
\hline & Richer & $2042(13.0)$ & $.995(.884)$, & $3.034(2.471,3.726) *$ \\
\hline & Richest & $5699(36.3)$ & $1.086(.968,1.219)$ & $3.462(2672,4.485) *$ \\
\hline \multirow[t]{3}{*}{ Frequency of watching TV } & Not at all & $10,084(64.3)$ & 1 & 1 \\
\hline & Less than once a week & $1780(11.3)$ & $.675(.618, .739)$ & $1.259(1.036,1.531)$ * \\
\hline & At least once a week & $3819(24.4)$ & $1.019(.895,1.161)$ & $1.368(1.110,1.687) *$ \\
\hline
\end{tabular}

${ }^{1}$ Reference

*Statistically significant

times more likely to utilize modern contraceptive method as compared to a woman who does not watch a television respectively (Table 4).

\section{Discussions}

The overall prevalence of modern contraceptive utilization among reproductive-age women in Ethiopia was 3203 (20.42\%). The finding is almost consistent with the result of the 2014 Ghana Demographic and Health Surveys secondary data analysis where the prevalence of modern contraceptive utilization was $21.53 \%$ [17]. It was also consistent with cross-sectional studies done in Ghana and Ethiopia in 2016 where 21 and $20.8 \%$ of reproductive age women used a modern contraceptive method respectively $[9,18]$. But the current prevalence is higher than the results of secondary data analysis of Nigeria (10.3\%) and Mali (15.3\%) Demographic and Health Survey which were conducted in 2013 and 2012 respectively [19, 20], and lower than the results of secondary data analysis of Burkina Faso (24\%) and Afghanistan (25.5\%) Demographic and Health Survey which were conducted in 2012 and 2015respectively [20, 21]. A cross-sectional multi-country analysis of Demographic and Health Surveys (DHS) conducted between 2008 and 2016 in 52 low and middleincome countries (LMICs) and taking 6857 representative sample from Ethiopia also showed that a prevalence of modern contraceptive method utilization among Ethiopian young reproductive-age women aged between 15 and 24 years was $12.1 \%$ which is by far lower than the result from the current study [15]. The current prevalence is also lower than the results of other studies done in Ethiopia at a different time [10-14]. The discrepancy might have occurred due to a difference in awareness of modern contraceptive methods. The difference might also be due to the socio-cultural difference between the different countries which might have a paramount effect on contraceptive utilization. The current study revealed that the injectable contraceptive method was the most commonly used contraceptive method which was consistent with the results of different studies done in Ethiopia [9, 11, 12, 14].

In the current study, age was an independent predictor of modern contraceptive method utilization among reproductive-age women in Ethiopia. A woman whose age was between 15 and 19, 20-24, 25-29, 30-34 and $35-39$ was nearly $1.9,2.4,2.2,1.9$ and 1.8 times more likely to utilize modern contraceptive methods as compared to a woman whose age was 40 year and above. Results from secondary data analysis of three consecutive Bangladesh Demographic and Health Surveys also showed that a woman whose age was from 40 to 49 years less likely practiced modern contraceptive utilization which is consistent with the current study [22]. The current result was also consistent with the results of two cross-sectional studies done in Northwest and Western Ethiopia where reproductive age women aged $35-49$ years and $>44$ years respectively were less likely utilized modern contraceptive method [10, 14]. Another cross-sectional study done in Cameroon in 2014 and 2015 also showed that age $>30$ years was negatively associated with modern contraceptive method utilization which was also consistent with the current study [8]. This might be due to the reason that reproductive-age women from low-income countries become more economically stable at their late reproductive age and might not want to utilize contraceptive methods to bear more children. But the current result is in contrast with the results of secondary data analysis of Nigeria (2013) and Afghanistan (2012) Demographic and Health survey where women greater than 40 years age more likely utilized modern contraceptive method [19, 23]. The 
difference might have occurred due to the difference in the socio-economic and socio-cultural differences between the countries.

Residence was also independently associated with modern contraceptive utilization in the current study. The odds of modern contraceptive utilization among urban resident reproductive-age woman was nearly 1.5 times more likely than the odds of modern contraceptive utilization among rural resident woman. The finding is consistent with the results of secondary data analysis of Indian, Afghanistan, Nigeria and Bangladesh Demographic and Health surveys where urban resident women more likely utilized modern contraceptive methods than rural resident women [19, 21, 22, 24]. This could be due to different reasons. Urban women are more educated, have better income, have better access to the health facility, and better media access than rural women which have a positive impact on modern contraceptive utilization. Rural women also need more children to help them with fieldwork which has a negative effect on their modern contraceptive method utilization $[14,19,21,23,25,26]$. But the result of secondary data analysis of the 2003-2014 Ghana Demographic and Health Surveys showed that rural resident women more likely utilized modern contraceptive methods than urban resident women which are in contrary to the results of the current study [17]. The difference could be due to the difference in awareness, availability, and accessibility of modern contraceptives for rural women.

The study also showed that the region of residence was an independent predictor of modern contraceptive method utilization among reproductive-age women. A woman who resides in Tigray, Amhara and SNNPR regions of Ethiopia was nearly 1.4,3 and 2 times more likely utilized modern contraceptive method as compared to a woman who resides in Addis Ababa respectively. A woman who resides in the Afar and Somali region of Ethiopia was nearly 55 and 93\% times less likely to utilize modern contraceptive methods as compared to a woman residing in Addis Ababa. The finding is consistent with the results of secondary data analysis of the 2013 Nigerian Demographic and Health Survey (NDHS) $[19,25]$ analyzed by independent researchers.

In the current study, the occupation was independently associated with modern contraceptive utilization. Reproductive age women who earn their lives by selling goods, agricultural employment, skilled manual working, and other different jobs were nearly 1.3, 1.2, 1.4 and 1.6 times more likely to utilize modern contraceptive methods as compared to reproductive-age women who did not have any job respectively. The finding is consistent with the finding from secondary data analysis of the 2003-2014 Ghana Demographic and Health Surveys [17]. The results of the descriptive study also depicted that $24.65,23.89,29.01$ and $24.44 \%$ of reproductive age women utilized modern contraceptive method from sales, agricultural employee, skilled manual working and others respectively, whereas, only $16.61 \%$ utilized modern contraceptive method from those who did not have any job. This could be due to the reason that women who are employed and have their private job do have better income, better access to media and health facilities which positively influence modern contraceptive method utilization $[14,19,21,23,25,26]$. Besides, they may need to have a gap between bearing children as they are on duty. Some women may be the only breadwinners for their families and might frequently work to sustain the lives of their families.

The number of children was also independently associated with the utilization of modern contraceptive methods. A woman who had no child and who had 1-2 child/children was nearly 75 and 34\% times less likely utilized modern contraceptive method as compared to a woman who had born 5 or more children. This finding is in line with the results of secondary data analysis of 2012 and 2015 Afghanistan Demographic and Health Survey where women with more than 6 children more likely utilized modern contraceptive methods [21, 23]. This may be due to the reason that women with a fewer number of children may need to bear more children to attain the desired family size [27]. But the current result is inconsistent with the result of secondary data analysis of three consecutive Bangladesh Demographic and Health Surveys where women with 4 or more children less likely utilized modern contraceptive methods as compared to women who had $0-1$ child [22]. The possible reason for this discrepancy could be the socioeconomic or socio-cultural difference between the two countries which might have an impact on the number of children desired. The result was also discordant with the result of the cross-sectional study done in Debre Birhan District of north Shoa zone, Ethiopia in 2010 where women who had more than five children less likely utilized modern contraceptive methods as compared to women who had no child [12]. This difference might have occurred due to the difference in the number of study participants participated in the study; the current study used a relatively large sample size which may better reflect the true population parameter as compared to any other study done with a small sample size.

The husband's educational status was positively associated with modern contraceptive method utilization. A woman whose husband had completed primary education was nearly 1.4 times more likely to utilize the modern contraceptive method as compared to a woman whose husband was not educated. The finding is consistent with the evidence from the 2014 Bangladesh Demographic and Health Survey where a woman whose 
husband completed higher education 1.28 times more likely utilized modern contraceptive methods as compared to a woman whose husband was not educated [28]. The finding was also concordant with the finding from a community-based cross-sectional study done at rural Dembia District, northwest Ethiopia in 2015 where a woman who had uneducated husband had $72 \%$ less likely utilized modern contraceptive method as compared to a woman who had a husband who completed grade 7 and above [10]. This could be due to the reason that educated husbands might have a good insight on modern contraceptives and compromise unreasonable social norms, beliefs, and attitudes towards modern contraceptive utilization and encourage their wives to utilize them. Besides, educated husbands may better share decision-making autonomy with their wives and approve the utilization of modern contraceptive utilization. Husband's education is also related to better household income which has a positive impact on modern contraceptive utilization $[14,19,26]$.

Multivariable logistic regression also showed that age at first sexual intercourse was independently associated with modern contraceptive utilization. A woman who had started her first sexual intercourse at an age of less than 18 years was nearly 1.6 times more likely to utilize the modern contraceptive method as compared to reproductive-age woman who started her first sexual intercourse at an age of 25 or more year respectively. This may be due to the reason that women under 18 years of age are, most of the time, economically dependent and do not want to bear a child and use a contraceptive to delay pregnancy. In the Ethiopian context, usually, reproductive age woman under 18 years of age wants to pursue her education and use a contraceptive to delay pregnancy. But this result is inconsistent with the result of the cross-sectional study done among sexually active Nepal youths, where those who started their first sexual intercourse between 12 and 15 years of age were less likely to use modern contraceptive as compared to those aged 20 to 24 year [29]. The difference could be due to the reason that the study done in Nepal considered only sexually active adult youths where the current study was done on all reproductive-age women aged 15 to 49 years.

The current study also revealed that the husband's desire for more children was inversely associated with modern contraceptive utilization. The odds of modern contraceptive utilization among women whose husbands want more children was nearly $25 \%$ less likely than the odds of modern contraceptive utilization among women who want the same number of children with their husbands. This is consistent with the evidence from Bangladesh, Burkina Faso and Mali secondary data analysis of Demographic and Health Survey where husband's desired number of children had influenced modern contraceptive utilization among reproductiveage women $[20,28]$. This could be due to male dominance in decision making autonomy in developing countries including our country, Ethiopia. When decisions are not equally made on the required family size in the household, males dominate the decision making power and decide on the number of the family size desired. Therefore, if husbands aspire for more children than their wives, they may negatively influence their spouses on utilizing family planning.

The current study also revealed that the wealth index was independently associated with current contraceptive utilization. A woman who was from the richest, a richer, middle and poorer household was nearly 3.5, 3, 2.5 and 2 times more likely to utilize modern contraceptive methods as compared to reproductive age woman from the poorest household respectively. This finding is consistent with findings from secondary data analysis of Demographic and Health Survey in Afghanistan in 2012 and 2015 [21, 23] and secondary data analysis of Demographic Health Survey in Nigeria in 2013 [19] where richest women more likely utilized modern contraceptive method as compared to poor women. The finding is also consistent with the results of a cross-sectional study conducted in Malawi in 2010 [26] and a cross-sectional study conducted in Nigeria in 2016 [25]. A crosssectional study conducted in western Ethiopia in 2014 also showed that a woman who had a monthly income of 1001-1500 ETB per month had nearly 2 times more likely utilized modern contraceptive methods as compared to a woman who earned less than 600EBR per month [14]. This might be due to the reason that the richest woman has more access to media and health facilities and might have a better awareness of modern contraceptive methods than those from a poor family $[14,19,26]$.

In the multivariable analysis, watching television was also independently associated with modern contraceptive utilization among reproductive-age women. A woman who watches television at least once a week and less than once a week was nearly 1.3 and 1.4 times more likely to utilize modern contraceptive methods as compared to a woman who does not watch television. The finding is consistent with the evidence from secondary data analysis of 2012 and 2015Afghanistan Demographic and Health Survey, 2014 Bangladesh Demographic and Health Survey and 2013 Nigeria Demographic and Health Survey where women who had media exposure utilized modern contraceptive method more likely as compared to women who had no media exposure $[21-23,25]$. This may be due to the reason that women who had media exposure might have a better awareness of modern contraceptives and their utilization. But a cross-sectional multi-country 
analysis of Demographic and Health Surveys (DHS) conducted between 2008 and 2016 in 52 low and middleincome countries (LMICs) showed that there is no association between exposure to media and modern contraceptive utilization among young reproductive-age women [15]. The difference might have occurred due to the age difference between the study participants; the current study included all reproductive-age women, whereas, the later one only included young reproductive-age women aged between 15 and 24 years. Young reproductive age women might not give due attention to the messages conveyed through media and benefitted from it.

The current study has its strengths and limitation. The study used nationally weighted representative data which better reflects the proportion of reproductive-age women using modern contraceptive method and its associated factors at the national level. Since the study used data from a single time survey, the temporal relationship between modern contraceptive use and the aforementioned predictor variables cannot be assured and the evidence should be utilized with caution. Besides, because of the lack of qualitative data on EDHS data, the association of qualitative variables like sociocultural factors to modern contraceptive utilization was not addressed in the current study.

In the current study, a woman was considered as modern contraceptive method utilizer if she had been using at least one of the modern contraceptives (female sterilization, male sterilization, IUCD, injectable, implants, pills, male condom, female condom, emergency contraception, and standard days method) only during EDHS data collection period. Therefore, since it was a single time survey, it does not show variations in the utilization of modern contraceptive methods by reproductive-age women in Ethiopia by period and results must be used with caution.

\section{Conclusions}

The magnitude of modern contraceptive utilization among reproductive-age women in Ethiopia in the current study is unexpectedly low. Age, residence, region, woman's occupation, number of living children, husband's education, age at first sexual intercourse, husband's desire for more children, wealth index and watching TV were independent predictors of modern contraceptive use among reproductive-age women in Ethiopia. Any intervention strategy that promotes modern contraceptive method utilization should consider these factors for its better success. Future researchers interested in the area should also address qualitative variables like socio-cultural factors which might have a tremendous effect on modern contraceptive utilization.

\section{Abbreviations}

AOR: Adjusted Odds Ratio; Cl: Confidence interval; COR: Crude Odds Ratio; CSA: Central Statistical Agency; EDHS: Ethiopia Demographic and Health Survey; ETB: Ethiopian Birr; FMoH: Federal Ministry of Health; LMIC: Low and Middle Income Country; SPSS: Statistical Package for Social Science

\section{Acknowledgments}

Our first and foremost gratitude goes to the Demographic and Health Survey (DHS) program for providing us 2016 EDHS data. We would also like to extend our deepest gratitude to Ethiopian Central Statistical Agency (CSA), Ethiopian federal ministry of health $(\mathrm{FMoH})$ and other stakeholders for their successful implementation of 2016 EDHS. Any governmental or non -governmental organization which directly or indirectly supported the 2016 EDHS data collection is also highly acknowledged. Finally, our heartfelt gratitude goes to all study participants, 2016 EDHS program coordinators, and fieldwork supervisors and data collectors.

\section{Authors' contributions}

MNG made a substantial contribution to designing the research concept, data acquisition, data processing, data analysis, report wring and manuscript drafting and revising. ZKE played a vital role in data processing, data analysis, and critical revision of the manuscript. Both authors read and approved the manuscript.

\section{Funding}

Not applicable.

\section{Availability of data and materials}

The SPSS datasets used and/or analyzed during the current study are available from the corresponding author on reasonable request.

\section{Ethics approval and consent to participate}

Permission to access the 2016 EDHS data was obtained from the DHS program by agreeing with the conditions of DHS data use stated on the $\mathrm{DHS}$ consent letter. Ethical clearance to conduct the survey was approved by the Ethical Review Board of Ethiopia Central Statistical Agency (CSA) and written informed consent was taken from study participants. Where participants are children (under 16 years old), written informed consent for participation in the study was obtained from their parent or guardian.

\section{Consent for publication}

Necessary permission for publication was obtained from DHS during data acquisition.

\section{Competing interests}

The authors declare that they have no competing interests.

Received: 15 October 2019 Accepted: 10 March 2020

Published online: 26 March 2020

\section{References}

1. Vlugt I Van Der. Contraceptives Knowledge file Index. 2018;(October).

2. Nations U. Family. new york; 2017.

3. WHO. Contraception fact sheet. Switzerland; 2012.

4. Mekonnen Y. A decade of change in contraceptive use in Ethiopia: in-depth analysis of the EDHS 200-2011. Addis Ababa; 2012

5. Hubacher D, Trussell J. A definition of modern contraceptive methods. ELSEVIER Contraception. 2015;92(5):420-1. Available from. https://doi.org/10. 1016/j.contraception.2015.08.008.

6. Access $\mathrm{O}$. Factors influencing use of modern contraception among married women in ho west district, Ghana: descriptive cross-sectional study. Pan Afr Med J. 2019;8688:1-11.

7. Ochako R, Askew I, Okal J, Oucho J, Temmerman M. Modern contraceptive use among migrant and non-migrant women in Kenya. BMC Reprod Health J. 2016:1-8. Available from. https://doi.org/10.1186/s12978-016-0183-3.

8. Njotang PN, Yakum MN, Ajong AB, Essi MJ, Akoh EW, Mesumbe NE, et al. Determinants of modern contraceptive practice in Yaoundé - Cameroon: a community-based cross-sectional study. BMC Res Notes J. 2017:4-9.

9. Semere Sileshi Belda, Mekonnen Tegegn Haile, Abulie Takele Melku and Abdurehaman Kalu Tololu. Modern contraceptive utilization and associated 
factors among married pastoralist women in Bale eco-region. BMC Health Serv Res. 2017;17(194):1-12.

10. Debebe S, Limenih MA, Biadgo B. Modern contraceptive methods utilization and associated factors among reproductive-aged women in rural Dembia District, northwest Ethiopia: Community based cross-sectional study. Int J Reprod BioMed. 2020;15(6):367-74.

11. Geremew AB, Gelagay AA. Modern contraceptive use and associated factors among married women in Finote Selam town Northwest Ethiopia : a community based cross-sectional study; 2018. p. 1-8.

12. Mohammed A, Woldeyohannes D, Feleke A, Megabiaw B. Determinants of modern contraceptive utilization among married women of reproductive age group in north Shoa Zone, Amhara Region, Ethiopia; 2014. p. 1-7.

13. Abraha TH, Teferra AS, Gelagay AA. Postpartum modern contraceptive use in northern Ethiopia : prevalence and associated factors; 2017. p. 1-9.

14. Tekelab T, Melka AS, Wirtu D. Predictors of modern contraceptive methods use among married women of reproductive age groups in Western Ethiopia : a community based cross-sectional study. BMC Womens Health. 2015:1-8. Available from:. https://doi.org/10.1186/s12905-015-0208-z.

15. Mutumba M, Wekesa E, Stephenson R. Community influences on modern contraceptive use among young women in low and middle-income countries : a cross- sectional multi-country analysis; 2018. p. 1-9.

16. ICF CSA (CSA) [Ethiopia]. Ethiopia. Addis Ababa and Rockville: CSA and ICF 2016.

17. Aviisah PA, Dery S, Atsu BK, Yawson A, Alotaibi RM, Rezk HR, et al. Modern contraceptive use among women of reproductive age in Ghana : analysis of the 2003-2014 Ghana Demographic and Health Surveys; 2018. p. 1-10.

18. Beson P, Appiah R, Adomah-Afari A. Modern contraceptive use among reproductive-aged women in Ghana: prevalence, predictors, and policy implications. BMC Womens Health. 2018;18(1):1-8.

19. Ofonime JE. Determinants of modern contraceptive uptake among Nigerian women: evidence from the national demographic and health survey. Afr J Reprod Health. 2017;21(3):89-95 Available from: https://www.scopus.com/ inward/record.uri?eid=2-s2.0-85038842247\&partnerlD=40\&md5=c36f097c56 e5413d445dcaaf7d7a30a1.

20. O'Regan A, Thompson G. Indicators of young women's modern contraceptive use in Burkina Faso and Mali from demographic and health survey data. Contracept Reprod Med. 2017;2(1):1-8.

21. Fort AL, Kothari MT, Abderrahim N. DHS Working Papers. Calverton Macro Int. 2008;(June): 1-47

22. Haq I, Sakib S, Talukder A. medical sciences Sociodemographic Factors on Contraceptive Use among Ever-Married Women of Reproductive Age : Evidence from Three Demographic and Health Surveys in Bangladesh; 2017.

23. Osmani AK, Reyer JA, Osmani AR, Hamajima N. Factors influencing contraceptive use among women in Afghanistan: secondary analysis of Afghanistan health survey 2012. Nagoya J Med Sci. 2015;77(4):551-61.

24. Dey AK. Socio-demographic determinants and modern family planning usage pattern-an analysis of National Family Health Survey-IV data. Int J Community Med Public Heal. 2019:6(2):738.

25. Adebowale AS, Gbadebo B, Afolabi FR. Wealth index, empowerment and modern contraceptive use among married women in Nigeria: are they interrelated? J Public Health. 2016;24(5):415-26. Available from:. https://doi org/10.1007/s10389-016-0738-3.

26. Adebowale SA, Adedini SA, Ibisomi LD, Palamuleni ME. Differential effects of wealth quintile on modern contraceptive use and fertility: Evidence from Malawian women. BMC Women's Health. 2014;14(1):2-13.

27. Matovu JKB, Makumbi F, Wanyenze RK, Serwadda D. Determinants of fertility desire among married or cohabiting individuals in Rakai, Uganda: A cross-sectional study. Reprod Health. 2017;14(1). Available from:). https://doi. org/10.1186/s12978-016-0272-3.

28. Hossain M, Khan M, Ababneh F, Shaw J. Identifying factors influencing contraceptive use in Bangladesh: evidence from BDHS 2014 data. BMC Public Health. 2018;18(1):1-14.

29. Tamang L, Raynes-Greenow C, McGeechan K, Black K. Factors associated with contraceptive use among sexually active Nepalese youths in the Kathmandu Valley. Contracept Reprod Med. 2017;2(1):1-8.

\section{Publisher's Note}

Springer Nature remains neutral with regard to jurisdictional claims in published maps and institutional affiliations.

\section{Ready to submit your research? Choose BMC and benefit from:}

- fast, convenient online submission

- thorough peer review by experienced researchers in your field

- rapid publication on acceptance

- support for research data, including large and complex data types

- gold Open Access which fosters wider collaboration and increased citations

- maximum visibility for your research: over $100 \mathrm{M}$ website views per year

At BMC, research is always in progress.

Learn more biomedcentral.com/submissions 